\title{
Collaborative Compressive Spectrum Sensing in a UAV Environment
}

\section{Citation}

Chen, Hsieh-Chung, H.T. Kung, Dario Vlah, Daniel Hague, Michael Muccio, and Brendon Poland. 2011. Collaborative compressive spectrum sensing in a UAV environment. In Proceedings of Military Communications Conference (MILCOM 2011), 142-148. Piscataway, New Jersey: IEEE Communications Society.

\section{Published Version}

doi:10.1109/MILCOM.2011.6127507

\section{Permanent link}

http://nrs.harvard.edu/urn-3:HUL.InstRepos:10054139

\section{Terms of Use}

This article was downloaded from Harvard University's DASH repository, and is made available under the terms and conditions applicable to Open Access Policy Articles, as set forth at http:// nrs.harvard.edu/urn-3:HUL.InstRepos:dash.current.terms-of-use\#OAP

\section{Share Your Story}

The Harvard community has made this article openly available.

Please share how this access benefits you. Submit a story.

\section{Accessibility}




\title{
Collaborative Compressive Spectrum Sensing in a UAV Environment
}

\author{
Hsieh-Chung Chen*, H. T. Kung*, Dario Vlah*, Daniel Hague ${ }^{\dagger}$, Michael Muccio $^{\dagger}$ and Brendon Poland ${ }^{\dagger}$ \\ * Harvard University, Cambridge, MA 02138 \\ $\dagger$ Air Force Research Laboratory, Rome, NY 13441
}

\begin{abstract}
Spectrum sensing is of fundamental importance to many wireless applications including cognitive radio channel assignment and radiolocation. However, conventional spectrum sensing can be prohibitively expensive in computation and network bandwidth when the bands under scanning are wide and highly contested. In this paper we propose distributed spectrum sensing with multiple sensing nodes in a UAV environment. The ground nodes in our scheme sense the spectrum in parallel using compressive sensing. Each sensor node transmits compressive measurements to a nearby UAV in the air. The UAV performs decoding on the received measurements; it decodes information with increasing resolution as it receives more measurements. Furthermore, by a property of compressive sensing decoding, frequencies of large magnitude responses are recovered first. In the proposed scheme, as soon as the UAV detects the presence of such high-power frequencies from a sensor, this information is used to aid decoding for other sensors. We argue that such collaboration enabled by UAV will greatly enhance the decoding accuracy of compressive sensing. We use packet-loss traces acquired in UAV flight experiments in the field, as well as field experiments involving software-defined radios, to validate the effectiveness of this distributed compressive sensing approach.
\end{abstract}

\section{INTRODUCTION}

Spectrum sensing is a widely used radio systems technique where certain portions of the spectrum are measured in order to discover RF emissions. There are numerous applications of spectrum sensing, of which we describe the following two prominent examples: cognitive radio and radiolocation.

In cognitive radio, devices attempt to find unused frequency bands to use for their own transmissions. It is expected that bands could be unused in spite of being licensed, or otherwise occupied in an official spectrum map. This can occur, e.g., if a license holder does not fully deploy across the licensed territory, or if licensing authorities or primary users insert guard bands into their spectrum (so called "white space"). Cognitive radios rely heavily on spectrum sensing to find the unused spectrum.

Radiolocation is a general problem where RF waves are used to determine the locations of certain objects of interest. Spectrum sensing is a particular way to perform radiolocation, where multiple sensors measure the spectrum at different points in a certain area; such readings can then lead to identification and trilateration of the detected transmitters.

In spite of the fact that most of the RF spectrum is licensed, studies [1] show that actual spectrum utilization is quite low. Furthermore, in target detection applications, the bandwidth occupied by targets of interest is often only a small fraction of the spectrum under measurement. As a result, a growing body of work focuses on performing the spectrum sensing using a technique called compressive sensing, notable for its ability to efficiently sample signals which are sparse in some basis. Using compressive sensing, it is possible to measure a frequency band of interest using many fewer samples than when sampling at Nyquist rate.

It is possible to distribute spectrum sensing, which is attractive for two main reasons. First, in applications such as radiolocation, spectrum measurements are needed from multiple vantage points to allow, e.g., triangulation based on time-difference-of-arrival (TDOA). Such measurements are naturally performed through multiple distributed sensors. Secondly, in cases where the spectrum bands of interest are wide, such as a $\mathrm{GHz}$ or more, speed of sensing can be much improved if the work is parallelized, so that multiple distributed sensors measure separate sub-bands concurrently.

In this paper, we combine distributed and compressive spectrum sensing, and enhance it by introducing collaboration. Specifically, we examine how we can improve the decoding of compressive measurements from one sensor by utilizing the decoded results from another. As we will see, the benefit of collaborative decoding can be substantial. We will refer to the resulting scheme as collaborative compressive spectrum sensing (CCSS).

We sketch the UAV environment of this paper in Figure 1. The UAV receives compressed measurements from ground sensors, and performs decoding for all of them. By leveraging its center role of knowing the decoded results for all ground sensors, the UAV implements collaborative decoding.

\section{BACKGROUND}

\section{A. Compressive sensing}

Compressive sensing has emerged as a major research area due to, among others, the surprising property that subNyquist sampling can capture the information present in a sparse signal. In general, this is made possible by having each measurement be some incoherent linear combination of the signal, thus ensuring that sparse signal components contribute to the sample with high probability.

A conventional compressive sensing encoding is formulated as follows:

$$
y=\boldsymbol{\Phi} x
$$



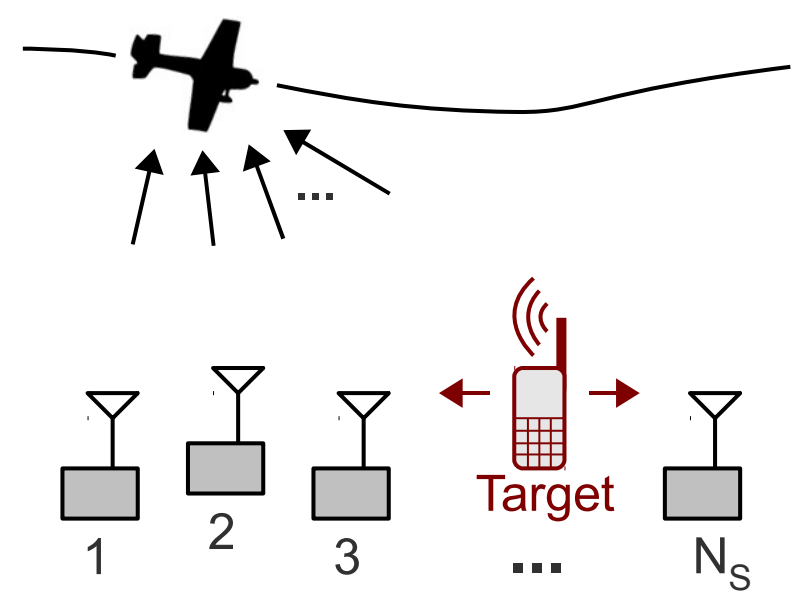

Fig. 1. The envisioned distributed spectrum sensing scenario. There are $N_{S}$ sensors measuring the spectrum in some area, potentially containing emitters, called targets, of interest, and send the measurements up to a UAV. The UAV performs further processing, such as weighted decoding on the data.

where $x$ is an $N$-dimensional vector representing the sparse signal being sampled, $\boldsymbol{\Phi}$ is an $M \times N$ measurement matrix containing random entries, and $y$ is a vector of $M$ measurements which are random linear combinations of components of $x$. Typically, $M \ll N$, so this is an underconstrained system that does not have a unique solution for general $x$. Nevertheless, suppose that $x$ is $K$-sparse in the sense that it can be expressed as a linear combination $\boldsymbol{\Psi} s$ of $K$ basis vectors in some basis $\boldsymbol{\Psi}$, using a vector $s$ of up to $K$ non-zero coefficients, and with $K$ being a fraction of $M$. Then it is possible to decode $x$ with high probability using a random $\boldsymbol{\Phi}$. A rich volume of literature examines this topic starting with the seminal work of Candès and Tao [2].

We adopt the conventional compressive sensing decoding method via $\ell_{1}$-minimization, by computing an approximation to the sparse vector $s$ as follows:

$$
\hat{s}=\underset{y=\mathbf{\Phi} \boldsymbol{\Psi} v}{\arg \min }\|v\|_{\ell_{1}}
$$

where $\boldsymbol{\Psi}$ is the transform basis that lets us express $x$ as a linear combination of its columns weighted by the sparse vector $s$. Once we obtain $\hat{s}$, it is straightforward to obtain an approximate $x$ :

$$
\hat{x}=\boldsymbol{\Psi} \hat{s}
$$

It has been shown that the $\ell_{1}$-reconstruction of sparse signals is exact with high probability if

$$
M>C K \log \frac{N}{K}
$$

for some small positive constant $C$ [3]. For example, in practice, $C=1.7$ with $\log _{2}$ gives a probability of decoding failure of less than $0.1 \%$.

\section{B. Weighted Decoding for Compressive Sensing}

It is possible to assist the process of decoding for compressive sensing by introducing weights into Equation (2) as follows:

$$
\hat{s}=\underset{y=\mathbf{\Phi} \boldsymbol{\Psi} v}{\arg \min }\left(w_{1}\left|v_{1}\right|+w_{2}\left|v_{2}\right|+\ldots+w_{N}\left|v_{N}\right|\right)
$$

Intuitively, weights $w_{i}>1$ increase the effect of a variable on the objective function, and so the minimization tends to avoid selecting those variables as one of the nonzero values in the solution. In the extreme case, suppose that with knowledge of the nonzero variables in the sparse input, we set $w_{i}=\infty$ for all the other variables; then the minimization will effectively operate on a subset of variables in an overconstrained linear system, making it trivial to solve. Weighting has been shown to be highly effective [4].

\section{Compressive Spectrum Sensing}

Compressive sensing is a natural way to sample wide spectrum bands, because usages of such bands are often sparse in the frequency domain. On the encoding side, we use Equation (1) with a fixed random matrix $\boldsymbol{\Phi}$ whose elements are independent random variables drawn from the normal distribution $\mathcal{N}(0,1)$, and the signal $x$ is a sequence of $N$ time domain samples of some spectrum band. On the decoding side, we use Equation (2) to obtain the sparse frequency domain coefficients by using an inverse discrete Fourier transform matrix for $\Psi$

\section{PROBLEM AND APPROACH}

Consider a scenario where $N_{S}$ sensors are distributed in a certain area, performing compressive spectrum sensing. Suppose we obtain $N_{S}$ sets of compressive measurements with one set from each sensor; we will refer to these measurements as measurement sets. As a baseline, we can decode the measurement sets individually. In collaborative decoding, however, we ask the question, how can we improve the baseline decoding results by collaborating on the decoded results from different measurement sets?

We will consider two main areas in which collaborative decoding can help:

- Sensor-diversity. Suppose signal strength of a particular emitter is weak at some sensor. The decoding for the signal from that emitter will be inaccurate. However, if the same emitter is received strongly at another sensor, the decoding results there could help improve the decoding accuracy for the weak reception.

- Measurement-delivery-variation. Consider a wireless transmission protocol for sensor measurements where the sensors transmit at a constant rate. It is likely that a mobile collection point, such as a nearby UAV, will receive varying numbers of measurements from the different sensors, depending on the link characteristics. In this case, we can improve the decoding accuracy for smaller measurement sets by decoding the larger measurement sets first, and using the results to guide the decoding of the smaller sets next.

Our overall approach consists of using the decoding results of one set of sensors to guide the decoding of another set. 
Specifically, we will use weighting, described in Section II-B, to modify the decoding process. In our two problem scenarios described above, we can use the initial decoding results to obtain weights to apply in weighted compressive decoding for subsequent results.

There are a number of challenges in using weighting for collaborative decoding, primarily related to parameter choice. First, the order in which we decode-and-weigh sensors could affect the outcomes, and needs to be evaluated. Secondly, having more than one iteration of collaboration may further improve the results; how many iterations is enough? Lastly, in order to actually perform the weighting, how do we compute the weights from the decoded results? We will address these questions in the following sections.

\section{Description of THE COllaborative DeCODing SCHEME}

In this section we describe our collaborative compressive spectrum sensing scheme. We start by describing the sampling process. Then, we present a basic scheme, called FixedCCSS, which processes the measurements with a fixed amount of collaboration. Lastly, we describe an enhanced scheme, Adaptive-CCSS, which processes the measurements according to the number of measurements received.

\section{A. Sampling process}

The sampling is performed by $N_{S}$ sensors shown in Figure 1. Each sensor obtains $N$ spectrum samples of the same band of interest centered at frequency $f_{C}$, and with bandwidth $B$. Let us write $x_{i}$ to denote the raw samples from sensor $i$. The sensors then compress the samples to $M$ compressive measurements by using a random $M \times N$ sampling matrix $\boldsymbol{\Phi}$, as described in Section II-A. Finally, each sensor transmits the $M$ samples to a collection point (i.e., the UAV in the application scenario of this paper) when prompted.

We assume that the wireless transmissions from the sensors are unreliable, so that fewer than $M$ measurements could arrive at the collection point from any given sensor. Let $y_{i}$ denote the vector of received measurements from sensor $i, M_{i}$ the size of vector $y_{i}$, with $M_{i} \leq M$, and $\boldsymbol{\Phi}_{\mathbf{i}}$ the appropriate submatrix of $\boldsymbol{\Phi}$ whose rows are used to compute elements of $y_{i}$. When no transmissions are lost from sensor $i$, we have $M_{i}=M$ and $\boldsymbol{\Phi}=\boldsymbol{\Phi}_{\mathbf{i}}$.

\section{B. Fixed-CCSS}

The basic scheme provides a way to decode measurement set, $\left\{y_{i}\right\}$, with a single round of collaborative correction. The scheme does not take into account the numbers of received equations, $\left\{M_{i}\right\}$, in the sense that the method for sensor $i$ does not adapt to the value of $M_{i}$. The scheme consists of five steps:

1) Decode each measurement set $y_{i}$ using standard compressive sensing decoding, obtaining solutions $\hat{x}_{i}$. Use an inverse discrete Fourier transform (IDFT) $\Psi$ matrix, so that the solutions $\hat{x}_{i}$ are in the frequency domain.
2) Sort elements of each vector $\hat{x}_{i}$ by magnitude, in decreasing order, obtaining a sorting order vector $u_{i}$. That is, the $k$-th element of vector $u_{i}$ is the index of the $k$-th largest-magnitude element in vector $\hat{x}_{i}$.

3) Truncate the sorting order vectors $u_{i}$ to the largest $\alpha$ entries, calling them $\bar{u}_{i}$, giving us the indices of the top magnitude elements of $\hat{x}_{i}$. Compute a union of all indices $\bar{u}_{i}$, denoting it as set $\mathcal{L}$. Note that the indices in $\mathcal{L}$ correspond to frequencies. For example, when $\alpha=1$, we take just the largest-magnitude frequency value from each sensor. Such values likely correspond to the strongest frequencies of the nearest targets to each sensor. Furthermore, when $\alpha=0$, no collaboration takes place and the results do not improve. The $\alpha$ value is fixed for all measurement sets, so we call the decoding scheme "Fixed." Furthermore, we will call the $\alpha$ value the collaboration gain for clarity.

4) For every index $j \in \mathcal{L}$ set weight $w(j):=1 / 10$. For all other indices $k \notin \mathcal{L}, w(k):=1$.

5) Finally, decode the measurements $y_{i}$ again, but this time using the weights $w(j)$ from Step 4.

\section{Adaptive-CCSS}

We extend Fixed-CCSS to take into account the amounts of the measurements received, as well as the magnitudes of the solutions, and term the resulting scheme Adaptive-CCSS. The main goal of the scheme is to lessen the influence of low-magnitude solutions or solutions computed from too few measurements. Specifically, the scheme determines a separate $\alpha$ value denoted $\alpha_{i}$ for each measurement set before Step 3, and then proceeds with weighted decoding in Steps 3-5 same as Fixed-CCSS. The method for determining the $\alpha_{i}$ for each measurement set works in the following two steps:

1) Denote the sorted elements of $\hat{x}_{i}$ as $u$. The sorting is by magnitude, in ascending order. Compute differences $v(j):=u(j+1)-u(j)$ for $j<N$.

2) Find smallest index $j$ such that $v(j)>C \cdot \operatorname{stdev}\left(y_{i}\right)$, where $C$ is a tunable parameter. Set $\alpha_{i}:=j$ if there exists such an index, or $\alpha_{i}:=0$ otherwise.

Intuitively, the method finds the distinguishing elements of $\hat{x}_{i}$ by looking for the sudden rise of magnitudes in the sorting of $\hat{x}_{i}$. To avoid finding such a rise in the noise floor we use the condition $v_{j}>C \cdot \operatorname{stdev}\left(y_{i}\right)$; note that $y_{i}$ are random projections of the input, and their statistics give us an indication of the noise level.

\section{Simulation Results}

In this section we evaluate the efficacy of the FixedCCSS and Adaptive-CCSS schemes in a spectrum sensing application using a simulated RF environment, and two types of measurement delivery: 1) over ideal links, with all sensors delivering the same number of measurements, and 2) over lossy links, where the loss rates were determined from traces of UAV links measured in the field. 


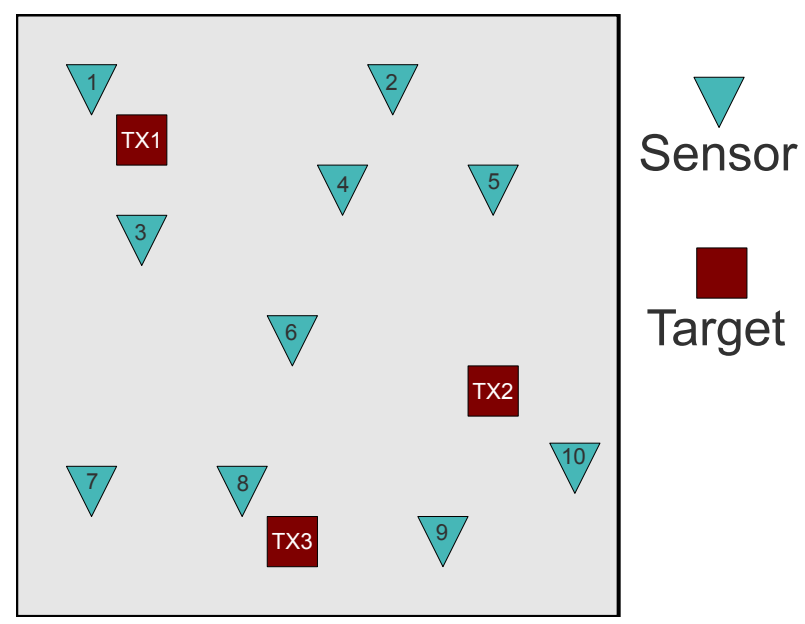

Fig. 2. Locations of sensors and targets (i.e., transmitters) in one example scenario.

\section{A. Experiment Setup}

We created a set of synthetic spectrum measurements by simulating signal propagation from $N_{T}$ transmitters to $N_{S}$ sensors, all located on a unit square region. Figure 2 depicts one instance of such a scenario with $N_{T}=3$ and $N_{S}=10$. In the experiments, we used $N_{T}=5$ and $N_{S}=5$.

We assumed that transmitter $T X_{i}$ output a continuous narrow-band signal at a distinct frequency $f_{i}$ with unit power. Thus, for the compressive sensing decoding process, we have the sparsity $K=N_{T}=5$. To compute propagation path loss, we used the free-space model. We drew the set of transmission frequencies $f_{i}$ at random without replacement from among $N / 2$ evenly spaced frequencies in the $200 \mathrm{MHz}$ band starting at $800 \mathrm{MHz}$, where we used $N=256$.

Each of the $N_{S}$ sensors samples the same $200 \mathrm{MHz}$ band. By taking $N$ real samples, the sensors obtain enough resolution to discern the $N / 2$ possible subchannels used by the targets. Complex samples can be handled by treating the real and imaginary parts as twice as many real samples. In addition, the spectrum samples are corrupted by noise; thus, the final time domain form of samples obtained at sensor $j$ is

$$
x(t)=\sum_{i=1}^{N_{T}} \frac{\mathrm{TX}_{\mathrm{i}}(t)}{D(i, j)^{2}}+z(t)
$$

where $\mathrm{TX}_{\mathrm{i}}(\mathrm{t})$ is the transmitted signal, $D(i, j)$ is the distance between target $i$ and sensor $j, z(t)$ is white Gaussian noise with zero mean and standard deviation 1 / SNR, and SNR is a parameter. In our simulations, we used $\mathrm{SNR}=10 \mathrm{~dB}$. Finally, we will write $x_{i}$ to denote the discrete sampled values of $x(t)$ for sensor $i$. The compressive measurements from each sensor are then

$$
y_{i}=\boldsymbol{\Phi} x_{i}
$$

where $\boldsymbol{\Phi}$ is an $M \times N$ measurement matrix whose entries are drawn independently from the standard normal distribution.

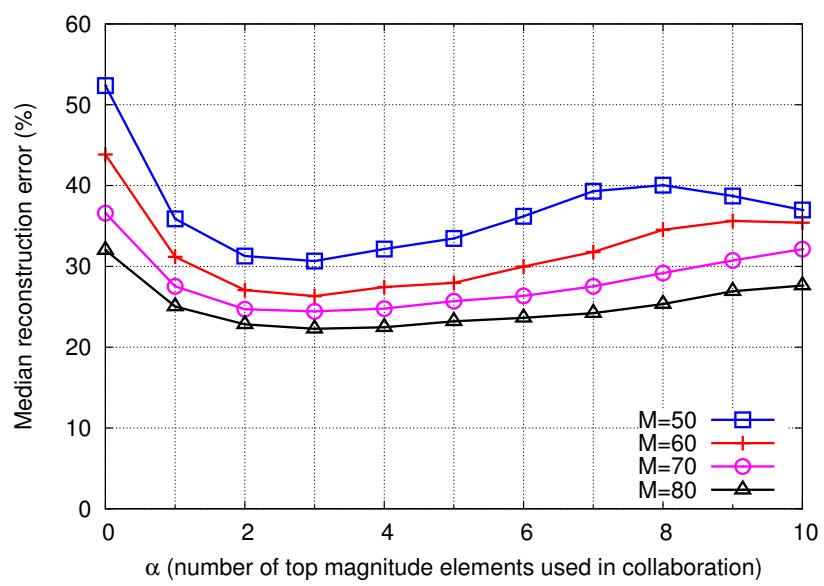

Fig. 3. Deviation of target frequencies from ground truth vs. the collaboration gain, $\alpha$.

\section{B. Results for Fixed-CCSS}

We present the performance of the Fixed-CCSS scheme over ideal links first. Figure 3 shows the effects of collaboration on decoding error of just the target frequencies. To control the "amount" of collaboration, we increase the collaboration gain $\alpha$ from 0 (no collaboration) to 10 (maximum collaboration). We perform the same decoding experiments for a range of values for the number of measurements $M$.

We can see that collaboration produces consistent improvements in decoding for small $\alpha$, regardless of the number of measurements. This confirms our expectation that weighting based on strong decoding results can help improve the weak ones. However, as $\alpha$ increases further, performance degrades; we can explain this by noting that the additional frequencies we weigh up are actually not target frequencies, but incorrectly identified ones, and thus increasingly dilute the solution.

In Figure 4 we present a different cross section of the same data, this time plotting error vs. number of measurements $M$. We plot two sets of results; one for a case without collaboration, and one for a case with $\alpha=2$, which seemed to be the best setting for $\alpha$ according to Figure 3. The main result evident in the plots is the dramatic reduction in the number of measurements needed to achieve the same error level; for example, the error without collaboration at $M=50$ is same as error with collaboration at roughly $M=30$, a $40 \%$ reduction in number of measurements.

\section{Simulation results for real-world UAV links}

In the previous section we performed the evaluation by assuming ideal sensor-to-data-collector links, that is, a fixed $M$ for all sensors. In this section, we consider a more realistic case when sensors have different $M$ s dictated by the quality of their link to the data collector. Specifically, we determine the $M$ values from field-measured traces of ground node-to-UAV packet transmissions.

In a previous publication [5] we reported in detail on a set of UAV field experiments where we measured the ground-to-UAV link qualities; we briefly summarize the experiments here. 


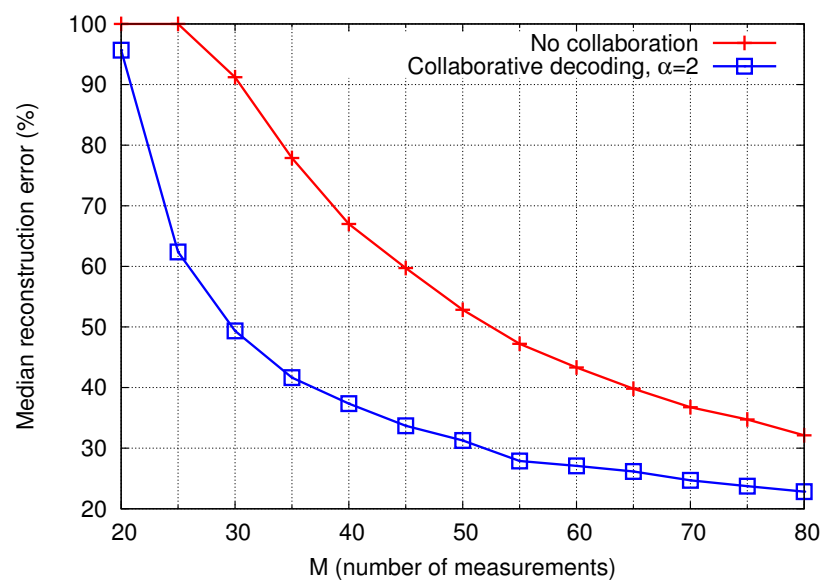

Fig. 4. Deviation of target frequencies from ground truth vs. number of measurements. Shown with and without collaboration.

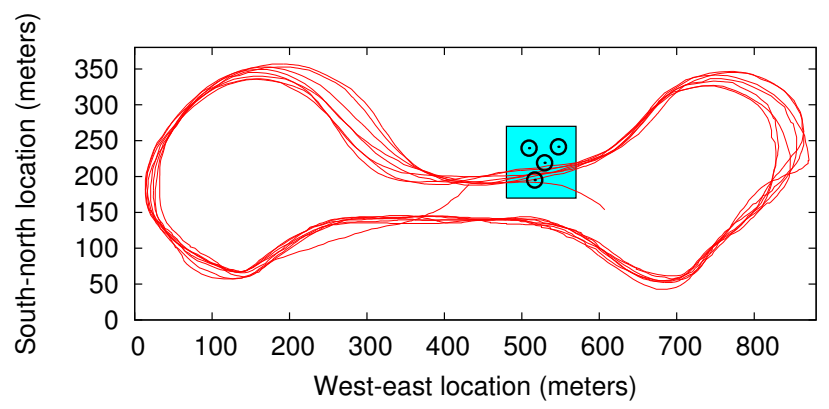

Fig. 5. UAV trajectory superimposed onto the ground target/sensor square.

Figure 5 shows the cyclic UAV trajectory traversed during one 16 minute flight. Also shown are the locations of 4 ground transmitter nodes. In terms of our spectrum sensing simulation, these 4 ground transmitters will be 4 sensors, which send data to the UAV. For the purpose of simulating the spectrum sensing targets, we will use a square region highlighted in the Figure, instead of a unit square of the previous section.

Figure 6 shows the link behavior of the 4 transmitters over time, for the entire 16 minute flight. We chose a time interval $T=2 \mathrm{~s}$, long enough to send $M=35$ measurements on a loss-free link; then, we divided the packet reception traces into blocks of duration $T$, and counted the number of packets successfully transmitted during each block. The results are shown in the Figure; furthermore, for clarity we show a small section of the flight in Figure 7, containing one flyover cycle. We can see that the receptions exhibit a periodic pattern, explained by the periodic nature of the flight path. Moreover, we can see that often all 4 transmitters have nonzero delivery rates to the UAV, such as in the right half of Figure 7; however, these delivery rates are generally not the same.

We present the UAV collaboration performance results in Figure 8, obtained using the Adaptive-CCSS scheme. As we can see from the data, collaboration brings about a consistent $\ell_{2}$ error improvement. Over the course of the 16 minute flight, the mean improvement was $65 \%$.

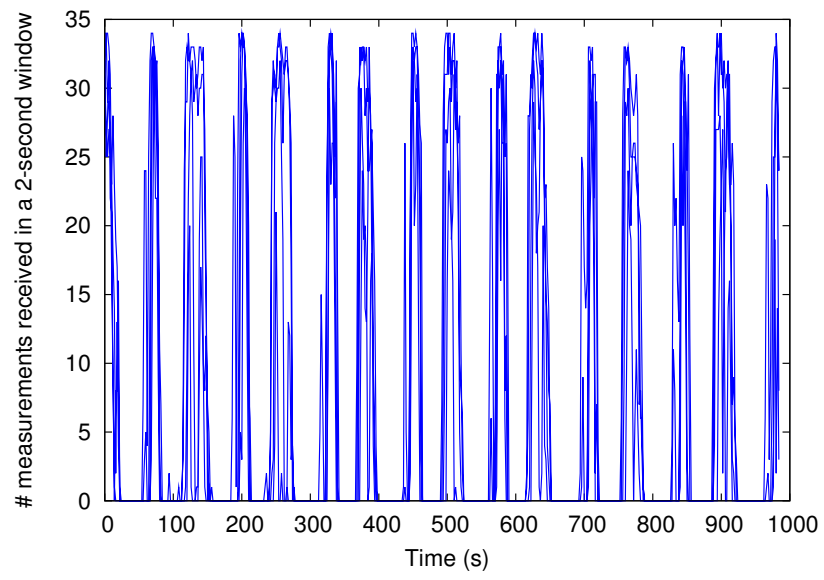

Fig. 6. Number of measurements received over time in 2 second intervals, computed from packet traces of an actual UAV flight. The numbers are shown for the four ground transmitters shown in Figure 5.

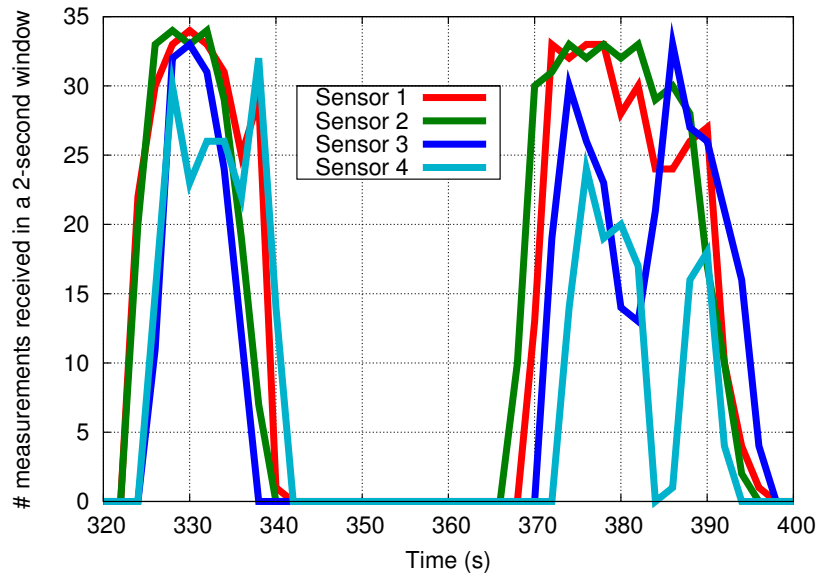

Fig. 7. Data from Figure 6 shown magnified to point out a basic data pattern of two peaks occuring during one UAV lap when the craft passes back and forth above the ground nodes.

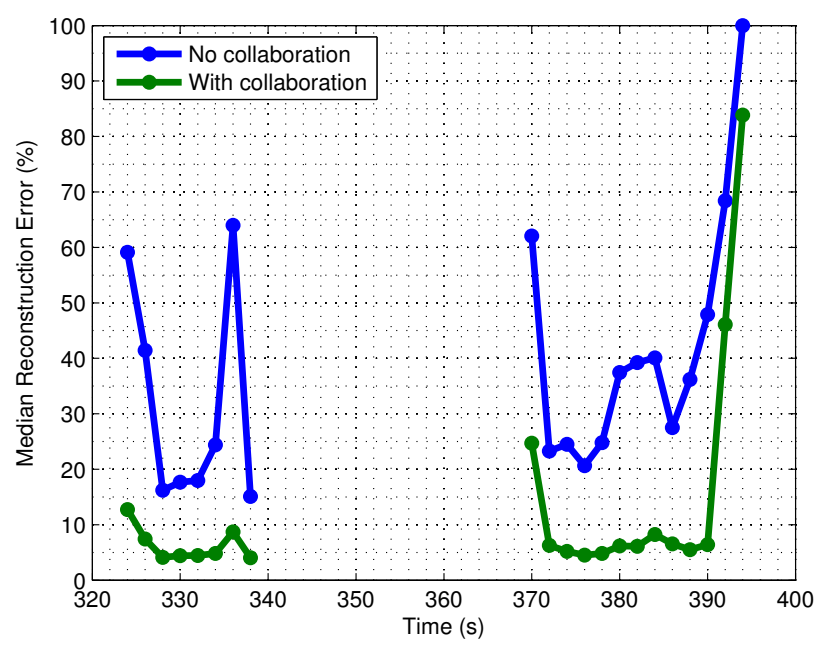

Fig. 8. Simulated performance gain of collaboration obtained using AdaptiveCCSS during one period of a cyclical UAV flight. The gaps in the data correspond to times when the craft is out of range of the ground transmitters. The portion of the flight used here is the same as that shown in Figure 7. 


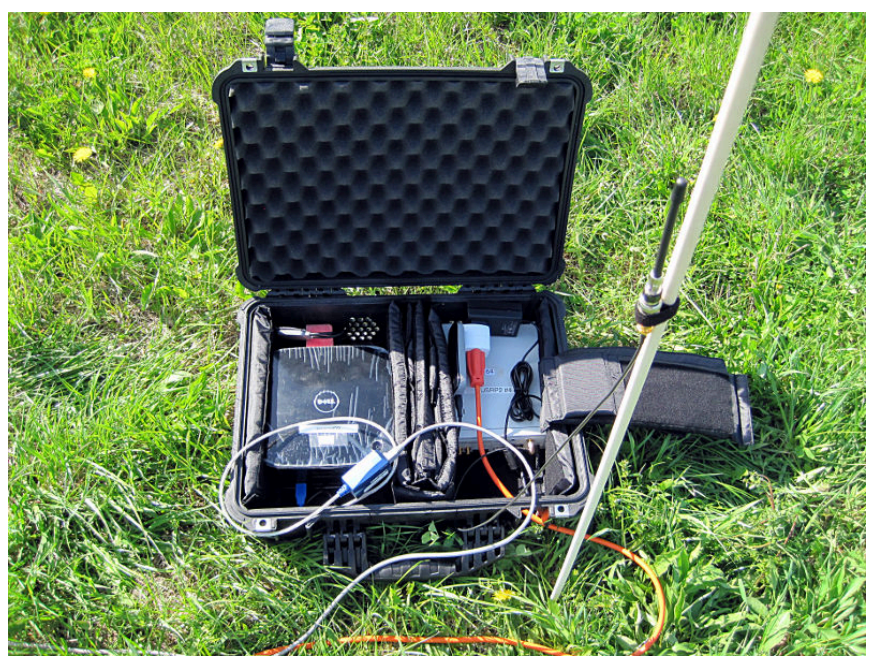

Fig. 9. GNURadio USRP2 sensor deployed in the field, along with the host PC used to record the measurements.

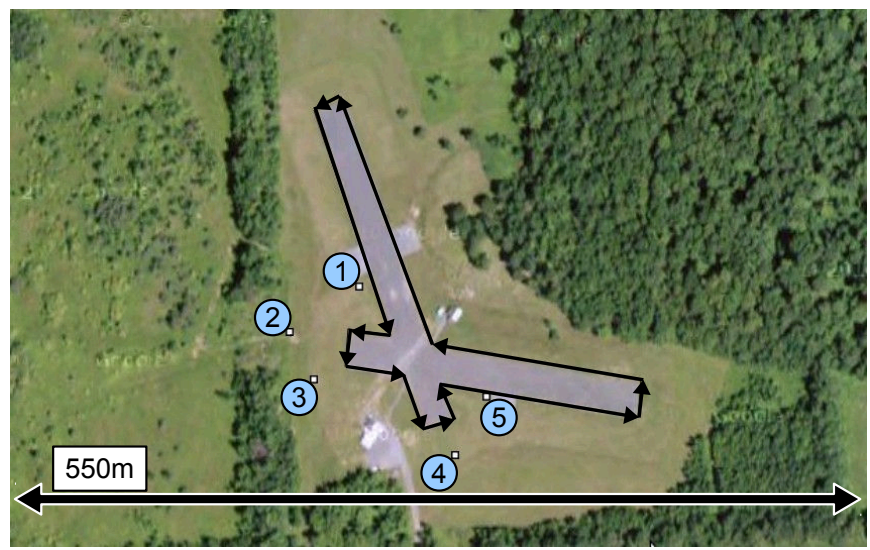

Fig. 10. Numbered circles indicate the locations of the 5 sensors deployed in the field. The arrows indicate the walking trajectory undertaken by the walkie-talkie operator.

\section{Field EXPERIMENT Results}

We conducted a spectrum sensing field experiment with 5 software radio sensors and one target. The sensors were GNUradio USRP2 devices with WBX daughterboards capable of sensing $25 \mathrm{MHz}$ bands within the $50 \mathrm{MHz}-2.2 \mathrm{GHz}$ range. One of the sensors is shown at its deployed location in Figure 9. Our target was a handheld walkie-talkie device transmitting at $5 \mathrm{~W}$ on the $370 \mathrm{MHz}$ carrier frequency, with most of the power concentrated in a band approximately $250 \mathrm{KHz}$ around the carrier.

The placement of the sensors is shown in Figure 10. The same Figure shows the path traveled by the transmitter, held by an operator while carrying on a conversation and walking at up to $200 \mathrm{~m}$ away from the sensors. The duration of the walk was roughly 10 minutes. Note that the operator's path created several cases where one sensor was particularly close to the transmitter; we have seen that such cases could be helped by collaborative decoding.

The GNURadio sensors recorded blocks of 256 complex

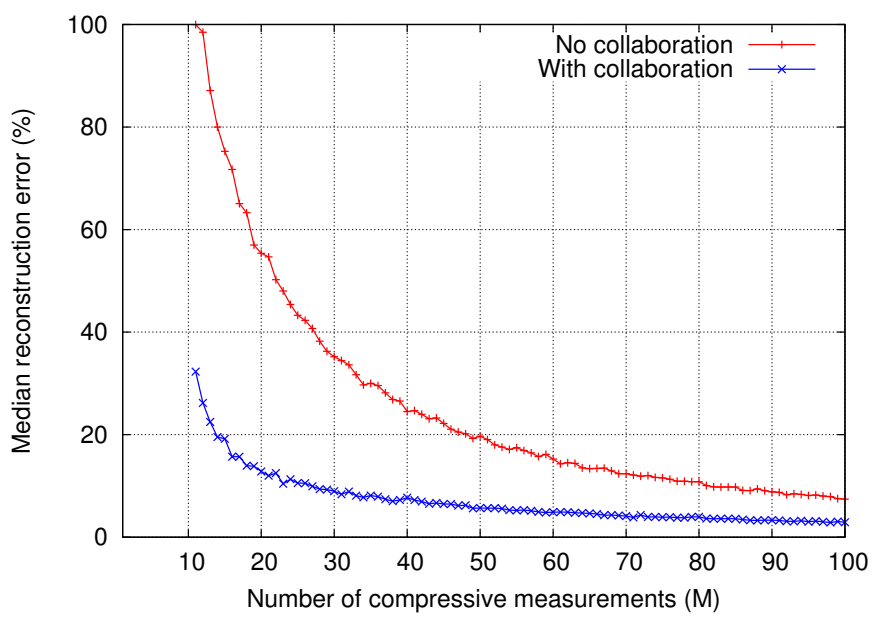

Fig. 11. Reconstruction accuracy of the signal strength of a walkie-talkie transmitter, achieved by 5 GNURadio sensors with and without collaboration, for an increasing number of compressive sensing measurements $M$.

samples at a time, sampled at the rate of $50 \mathrm{MHz}$ at the center frequency of $373 \mathrm{MHz}$. One such block was recorded every $100 \mathrm{~ms}$, giving a total of about 6,000 blocks in our 10 minute experiment. Each block was used as the input for one instance of compressive sensing, so that after expanding each complex sample as a pair of real numbers, $N=512$. With one transmitter active, its transmit power was observed in 5 adjacent FFT coefficients, so the sparsity $K$ was approximately 5. However, there were other active transmissions in our band of interest, so the sparsity turned out to be somewhat highermainly from 5 to approximately 15 .

\section{A. Reconstruction accuracy vs. number of measurements $M$}

We examine the reconstruction accuracy as a function of the number of compressive measurements $M$. We varied $M$ from 10 to 100; at the upper limit, the number of measurements represents approximately $1 / 5$ of the original samples. We decoded each compressive sensing instance with and without collaboration. In both cases, for each $M$, we computed the reconstruction error of the magnitude of FFT coefficients at the target frequencies as a percentage difference relative to the ground truth magnitude. Each data point is a median of 100 runs. Figure 11 shows the resulting reconstruction accuracy over increasing $M$. We can see that the effect of collaboration is significant for any $M$ value; on average, the median reconstruction error was reduced by $69 \%$.

\section{RELATED WORK}

Researchers have considered the problem of collaborative compressive sensing before. In Meng et al. [6] the authors consider a similar spectrum sensing problem as ours; to solve it, they adapt an existing matrix completion algorithm to perform joint-sparsity reconstruction. This work differs from our paper in that we do not use matrix completion, but instead modify the conventional compressive sensing decoding process to incorporate information from external nodes. Furthermore, the 
authors only evaluated a noiseless case, whereas our evaluation considers noise.

Finally, we note that in [7], a weighted decoding scheme similar to ours is used to mitigate blocking artifacts resulting from partitioned compressive sensing. In [8], compressive sensing for distributed sensor systems is studied for a different set of issues, that is, in-network measurement combining and progressive decoding to reduce both measurement and decoding costs.

\section{CONCLUSION}

In this paper, we presented simple collaborative decoding schemes for compressive spectrum sensing. Specifically, our scheme improves the decoding of compressive measurements from one sensor by utilizing the decoded results from another. We have shown that the benefits of such collaborative decoding can be substantial.

We applied the collaborative compressive spectrum sensing in a UAV scenario, where the UAV serves as a natural collection point for the distributed measurements. We have shown that our decoding scheme is well adapted for this scenario because it easily tolerates wireless transmission losses; thanks to the properties of compressive measurements, only the amount of measurements is important, not their precise identity.

In terms of applications, results of this paper mean faster and more accurate sensing of ground targets by UAV. As future work, we plan to explore use of collaborative compressive sensing in localization of ground targets and other applications.

\section{ACKNOWLEDGEMENTS}

This material is based on research sponsored by Air Force Research Laboratory under agreement number FA8750-10-20180. The U.S. Government is authorized to reproduce and distribute reprints for Governmental purposes notwithstanding any copyright notation thereon. The views and conclusions contained herein are those of the authors and should not be interpreted as necessarily representing the official policies or endorsements, either expressed or implied, of Air Force Research Laboratory or the U.S. Government. The authors would like to thank the Office of the Secretary of Defense (OSD/ASD(R\&E)/RD/IS\&CS) for their guidance and support of this research.

\section{REFERENCES}

[1] M. A. McHenry, P. A. Tenhula, D. McCloskey, D. A. Roberson, and C. S. Hood, "Chicago spectrum occupancy measurements \& analysis and a long-term studies proposal," in Proceedings of the first international workshop on technology and policy for accessing spectrum (TAPAS '06), Boston, MA, USA, Aug. 2006.

[2] E. J. Candès and T. Tao, "Decoding by linear programming," IEEE Trans. Inf. Theory, vol. 51, no. 12, pp. 4203-4215, Dec. 2005.

[3] E. J. Candès, J. K. Romberg, and T. Tao, "Robust uncertainty principles: Exact signal reconstruction from highly incomplete frequency information," IEEE Trans. Inf. Theory, vol. 52, no. 2, pp. 489-509, 2006.

[4] E. J. Candès, M. B. Wakin, and S. P. Boyd, "Improving the performance of reliable transport protocols in mobile computing environments," Journal of Fourier Analysis and Applications, special issue on sparsity, vol. 14, no. 5, pp. 877-905, Dec. 2008.
[5] H. T. Kung, C.-K. Lin, T.-H. Lin, S. Tarsa, and D. Vlah, "Measuring Receiver Diversity on a Low-Altitude UAV in a Ground-to-Air Wireless Mesh Network," in International Workshop on Wireless Networking for Unmanned Aerial Vehicles (Wi-UAV 2010), Miami, FL, USA, Dec. 2010.

[6] J. Meng, W. Yin, H. Li, E. Houssain, and Z. Han, "Collaborative spectrum sensing from sparse observations using matrix completion for cognitive radio networks," in ICASSP 2010, Dallas, TX, USA, Mar. 2010.

[7] H. T. Kung and S. Tarsa, "Partitioned compressive sensing with neighborweighted decoding," MILCOM, 2011.

[8] H.-C. Chen, H. T. Kung, D. Vlah, and B. Suter, "Measurement combining and progressive reconstruction in compressive sensing," MILCOM, 2011. 